Focussed on "Crack Paths"

\title{
Crack paths in soft thin sheets
}

\author{
Roberto Brighenti, Andrea Carpinteri, Federico Artoni \\ Department of Engineering and Architecture, University of Parma, Viale Usberti 181/A, 43124 Parma, Italy \\ brigh@unipr.it,bttps:/ /orcid.org/0000-0002-9273-0822 \\ andrea.carpinteri@unitpr.it, bttps:/ / orcid.org/0000-0002-8489-6005 \\ federico.artoni@studenti.unipr.it, bttps:/ /orcid.org/0000-0001-9032-2959
}

ABSTRACT. Highly deformable materials (elastomers, gels, biological tissues, etc.) are ubiquitous in nature as well as in technology. The understanding of their flaw sensitivity is crucial to ensure a desired safety level. Fracture failure in soft materials usually occurs after the development of an uncommon crack path because of the non-classical near-tip stress field and the viscous effects. In a neo-Hookean material, the true opening stress singularity along the crack path (evaluated normal to the crack line) is of the order $r^{-2}$, while it is of the order $s^{-1 / 2}$ ahead of the crack tip, promoting the appearance of a crack tip splitting leading to a tortuous crack. In the present paper, experimental tests concerning the fracture behavior of highly deformable thin sheets under tension are discussed, and the observed crack paths are interpreted according to the crack tip stress field arising for large deformations. The study reveals that higher strain rates facilitate the development of a simple Mode I crack path, while lower strain rates induce a mixed Mode in the first crack propagation stage, leading to the formation of new crack tips. The above described behavior seems to not be affected by the initial crack size.

KEYwORDS. Rubber; Highly deformable materials; Crack path; Strain rate; Crack tip splitting.

\section{open ACCESS}

Citation: Brighenti, R., Carpinteri, A., Artoni, F., Crack paths in soft thin sheets, Frattura ed Integrità Strutturale, 48 (2019) 1 -9.

Received: 15.10 .2018

Accepted: 06.01.2019

Published: 01.04.2019

Copyright: (C) 2019 This is an open access article under the terms of the CC-BY 4.0, which permits unrestricted use, distribution, and reproduction in any medium, provided the original author and source are credited.

\section{INTRODUCTION}

S oft materials such as rubbers, elastomers, gels, foams, granular and many biological materials are usually prone to easily deform, leading to a highly nonlinear response under mechanical actions [1,2]. On the other hand, the capability to modulate their toughness or viscosity has promoted a wide attention in advanced applications (from materials science to bioengineering) where such materials can be conveniently exploited to get smart and functional materials such as artificial muscles, active and self-morphing materials $[3,4]$.

Furthermore, highly deformable materials often exhibit a rate-dependent response [5, 6], and in some cases (such as for natural rubbers) when sufficiently stretched at room temperature, show a strain-induced crystallization inducing a change 
from an amorphous into a semicrystalline-like microstructural configuration due to the appearance of a highly oriented microstructure developing along the tensile direction [7].

The microstructure of polymer materials is fully amorphous and, at the molecular level, is formed by a three-dimensional network of polymer chains linked at several discrete points (cross-links). The mechanics of these materials depends on the relative interaction and motion of the entangled linear macromolecules; the physical-chemistry basis of such a class of materials has been firstly established by Paul J. Flory, P.G. de Gennes and L.R.G. Treloar [8-10], through their fundamental theoretical and experimental research work.

Another key aspect of soft materials is their ability to withstand defects (such as cracks and notches) in presence of mechanical actions, without showing brittle failure [11, 12]; in the near tip region of a crack, the local large deformation induces a microstructure rearrangement (chains alignment) and promotes a change in the macroscopic shape of the defect. These mechanisms enhance a material strengthening-like mechanism, leading to a noticeable defect tolerance capability. In the present study, we consider the fracture behavior of highly deformable thin cracked sheets under tension. Experimental tests performed at various strain rates on pre-cracked soft rubber specimens are presented, and the experimentally observed crack paths are interpreted according to the crack tip stress field arising in the case of large deformations. The experimental outcomes show that, for cracks tested in Mode I, higher strain rates facilitate the development of a simple Mode I crack path, while lower strain rates can induce a mixed Mode in the first stage of the crack propagation. Moreover, during the loading process the appearance of new crack tips due to the Mode mixity has been observed as a consequence of the nonclassical stress field existing around the crack tip. Tests have confirmed that the above described mechanical response in terms of crack path is not influenced by the initial crack size.

\section{CRACK TIP STRESS FIELD IN HIGHLY DEFORMABLE MATERIALS}

A typical way to measure the flaw tolerance of materials is through the determination of the fracture energy; experiments conducted on soft polymers indicated a strong rate and temperature dependency for such a fracture property because of the energy dissipation produced by viscoelasticity [13].

Some different approaches to explain the failure in rubber-like materials have been proposed; among them the so-called cavitation criterion assumes the presence of intrinsic small defects that lead to failure under a sufficiently large hydrostatic stress state provoking void expansion and coalescence [14].

On the other hand, within the classical fracture mechanics approach, the near crack tip stress field governs the local failure of the material in terms of chains failure and growth of small existing microdefects and voids. Because of the high deformation capability of this class of materials, the classical Linear Elastic Fracture Mechanics (LEFM) approach - valid for infinitesimal strains - is not applicable anymore, and the large displacement effects must be accounted for instead. Further, the isochoric (incompressible) deformation process, typically shown by polymers, must be also considered in the determination of the singular stress field induced by a crack $[15,16]$.

In Fig. 1 the geometry of the considered cracked plate is shown; the global reference coordinate system $X, Y$ and the crack tip reference coordinates $x_{1}, x_{2}$ are depicted in Fig. 1a, while the current (deformed) crack tip coordinates are indicated with $y_{1}, y_{2}$ (Fig. 1b). Note that the crack tip moves from the initial position $o$ to the current one $o^{\prime}$.

\section{Formulation of the problem in large deformation}

In the reference coordinate system $x_{1}, x_{2}$, the equilibrium equations in absence of body forces and the traction-free boundary conditions are

$$
\partial P_{i j} / \partial x_{j}=0, i, j=1,2, \quad P_{22}(r>0, \theta= \pm \pi)=P_{12}(r>0, \theta= \pm \pi)=0
$$

where the nominal stress tensor $\boldsymbol{P}$ (Piola stress) has been used, while the true stress referred to the current deformed configuration (Cauchy stress $\boldsymbol{\sigma}$ ) is related to the nominal Piola stress through the relationship $\boldsymbol{\sigma}=J^{-1} \boldsymbol{P F}^{T}$, $\boldsymbol{F}=F_{i j}=\delta_{i j}+\partial u_{i} / \partial x_{j}$ being the deformation gradient tensor of the displacement $u_{i}$ and $J=\operatorname{det} \boldsymbol{F}$ being the volume variation which, for an incompressible material, must be equal to 1 . 

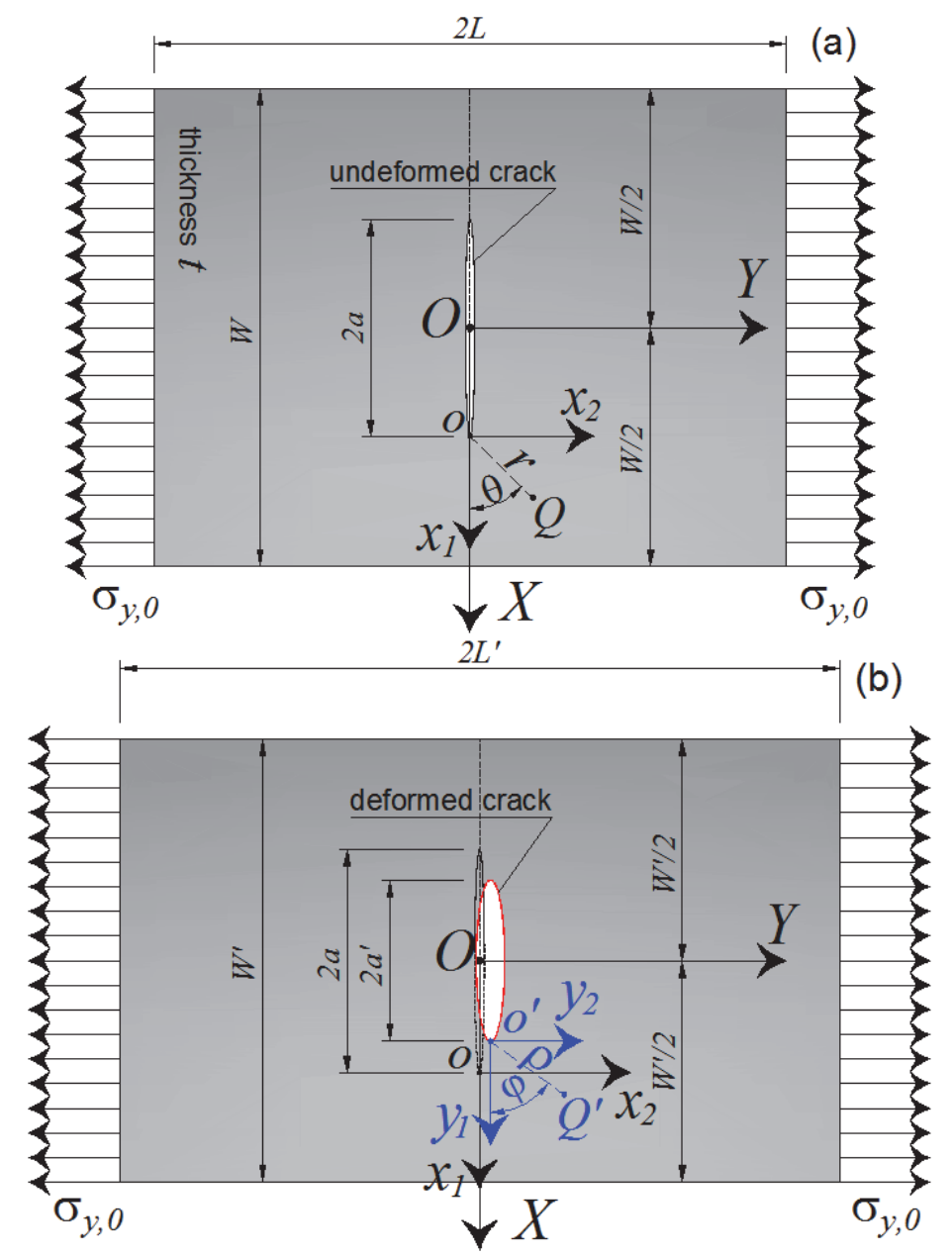

Figure 1: Cracked plate: main geometrical sizes and reference systems in the (a) undeformed and (b) deformed configuration.

\section{Constitutive models}

Constitutive models for polymers are usually defined in terms of the strain energy function $\hat{\Psi}$; several models suitable for rubber-like materials have been proposed in the literature. Among them, the well-known neo-Hookean, Gent and Ogden models can be mentioned [17]. In particular, the following energy expression $\psi(r)$ for the deformation of a single chain is assumed by the neo-Hookean model, and the strain energy density follows the Gent model, respectively:

$$
\psi(r)=\frac{3 k_{B} T}{2 N b^{2}} \cdot r^{2}, \quad \hat{\Psi}\left(J_{1}\right)=\frac{E}{6} J_{m} \ln \left(1-\frac{J_{1}}{J_{m}}\right)
$$

where $k_{B}$ is the Boltzmann constant and $T$ the absolute temperature. Further, $N$ is the number of Kuhn's segments in a single polymer chain and $b$ is their length, whereas $E$ is the small strain Young modulus, $J_{1}=I_{1}-3$ is a stretch invariant, and $J_{m}$ is the limit value of $J_{1}$. The energy per unit volume of the material $\Psi$ can be obtained from the following relationship:

$$
\hat{\Psi}=\int_{\Omega} \phi(\boldsymbol{r}) \psi(\boldsymbol{r}) d \Omega
$$


where $\Omega=\left\{\boldsymbol{r} \in \mathbb{R}^{3}\right\}$ indicates the chain configuration space and $\phi(\boldsymbol{r})$ is the chains' end-to-end distance distribution function, i.e. it provides the number of chains per unit volume whose end-to-end distance is comprised between $\boldsymbol{r}$ and $\boldsymbol{r}+d \boldsymbol{r}$. Once the energy function is known, the nominal stress tensor can be obtained from the strain energy as

$$
\boldsymbol{P}=\frac{\partial \hat{\Psi}}{\partial \boldsymbol{F}}=\frac{\partial}{\partial \boldsymbol{F}} \int_{\Omega} \phi(r) \psi(r) d \Omega+p(t) J \boldsymbol{F}^{-T}
$$

where $p$ is the hydrostatic pressure, introduced as a Lagrange multiplier to enforce the incompressibility condition herein assumed for the polymer as usually observed for this class of materials, being $J=\operatorname{det} \boldsymbol{F}=1$.

The above expression(3), in the case of the standard Gaussian distribution $\phi(r)$ of $r$ (typically adopted in rubber elasticity), leads to $\Psi=\mu\left(I_{1}-3\right) / 2$, where $I_{1}=\lambda_{1}^{2}+\lambda_{2}^{2}+\lambda_{3}^{2}$, is the first invariant of the right Cauchy-Green deformation tensor $\boldsymbol{C}=\boldsymbol{F}^{T} \boldsymbol{F}, \mu$ is the small strain shear modulus and $\lambda_{1}, \lambda_{2}, \lambda_{3}$ are the three stretches along the Cartesian directions. An energy expression valid for large strains of a single chain $\psi$ is the one proposed by Kuhn, namely $\psi(r)=N k_{B} T \cdot\left(\frac{\beta}{b N} r+\ln \frac{\beta}{\sinh \beta}\right)$, where $\beta=\mathcal{L}^{-1}\left(\frac{r}{b N}\right)=\mathcal{L}^{-1}\left(\frac{\lambda}{\sqrt{N}}\right), \mathcal{L}^{-1}$ is the inverse Langevin function, while $b, N$ and $\lambda$ are the Kuhn's segment length, the number of segments per chain and the chain stretch, respectively.

\section{Crack tip stress field}

It is well-known that, in a 2D problem, the Mode I crack tip stress field within the LEFM hypothesis is expressed by:

$$
P_{i j}=\sigma_{i j}=\frac{K_{\mathrm{I}}}{\sqrt{2 \pi r}} f_{i j}(\theta)+\text { b.o.t. }, i, j=1,2
$$

where $f_{i j}(\theta)$ is an angular function, $K_{\mathrm{I}}$ is the Mode I Stress-Intensity Factor (SIF) and, thanks to the small deformation hypothesis, the nominal $\left(P_{i j}\right)$ and true stress $\left(\sigma_{i j}\right)$ tensor components are identical. Within the LEFM, the stresses are linear function of strain, and can be superimposed. Further, all the stress components have the same inverse square root singularity for the three deformation modes (Mode I, Mode II and Mode III).

Within the large displacement field context, Knowles and Sternberg [18] performed the crack-tip asymptotic analysis through a series expansion of the deformed coordinates, consisting of separable functions of the polar coordinates $(r, \theta)$ in the undeformed material, in a way similar to the William's expansion approach in LEFM [19].

The crack tip stress field under large deformation results to be quite different from the one valid in the LEFM hypothesis and depends upon the constitutive model in turn; such a problem has been solved applying the asymptotic analysis method [20] by writing the crack tip deformed coordinates, $y_{1}, y_{2}$, with respect to the undeformed material coordinate system, $x_{1}, x_{2}$, through a series of separable functions of the polar coordinates $r, \theta$ (Fig. 1). It can be shown that, under large deformation the true (Cauchy) stress components for a neo-Hookean incompressible material are given by

$$
\sigma_{11}=\mu C^{2}, \sigma_{12}(r, \theta)=\sigma_{21}=-\frac{\mu}{2} A C r^{-1 / 2} \sin \frac{\theta}{2}, \sigma_{22}(r, \theta)=\frac{\mu}{4} A^{2} r^{-1}
$$

where $A, C$ are unknown positive amplitudes depending on loading and geometry of the configuration being examined, and the pressure field required to enforce the incompressibility condition (usually assumed for polymeric materials since they can undergo only isochoric deformations) turns out to be $p=-2 \mu C A^{-1} r^{1 / 2} \cos \frac{\theta}{2}$. The stress field is thus governed by these two parameters instead of the only one (the SIF, $K_{\mathrm{I}}$ ) needed in the LEFM.

From Eq. (6) it is evident that, differently from the LEFM case, the singularity of the opening stress component $\sigma_{22}$ is different from the singularity of the shear stress component $\sigma_{12}$ as $r$ approaches the crack tip. 
The knowledge of the kinematic relationship between deformed and reference configuration, $y_{1}=\operatorname{cr} \cos \theta, y_{2}=a \sqrt{r} \sin \theta / 2$ [20], allows to express the true stress components in terms of the polar coordinates $(\rho, \varphi)$ in the deformed (current) configuration (Fig. 1b, Fig. 2).

Along the deformed upper parabolic crack profile, the true stress components are:

$$
\sigma_{11}=\mu C^{2}, \sigma_{12}=\sigma_{21}\left(\rho, \varphi=\frac{\pi}{2}\right)=-\frac{\mu}{4} A^{2} C \rho^{-1}, \sigma_{22}\left(\rho, \varphi=\frac{\pi}{2}\right)=\frac{\mu}{8} A^{4} \rho^{-2}, \rho \rightarrow 0
$$

where only the singular terms have been reported and the relationship $\varphi=\theta / 2$ has been considered due to the fact that the deformed crack profile at the crack tip becomes parallel to the vertical axis (the tangent to the parabolic profile is vertical) [20].

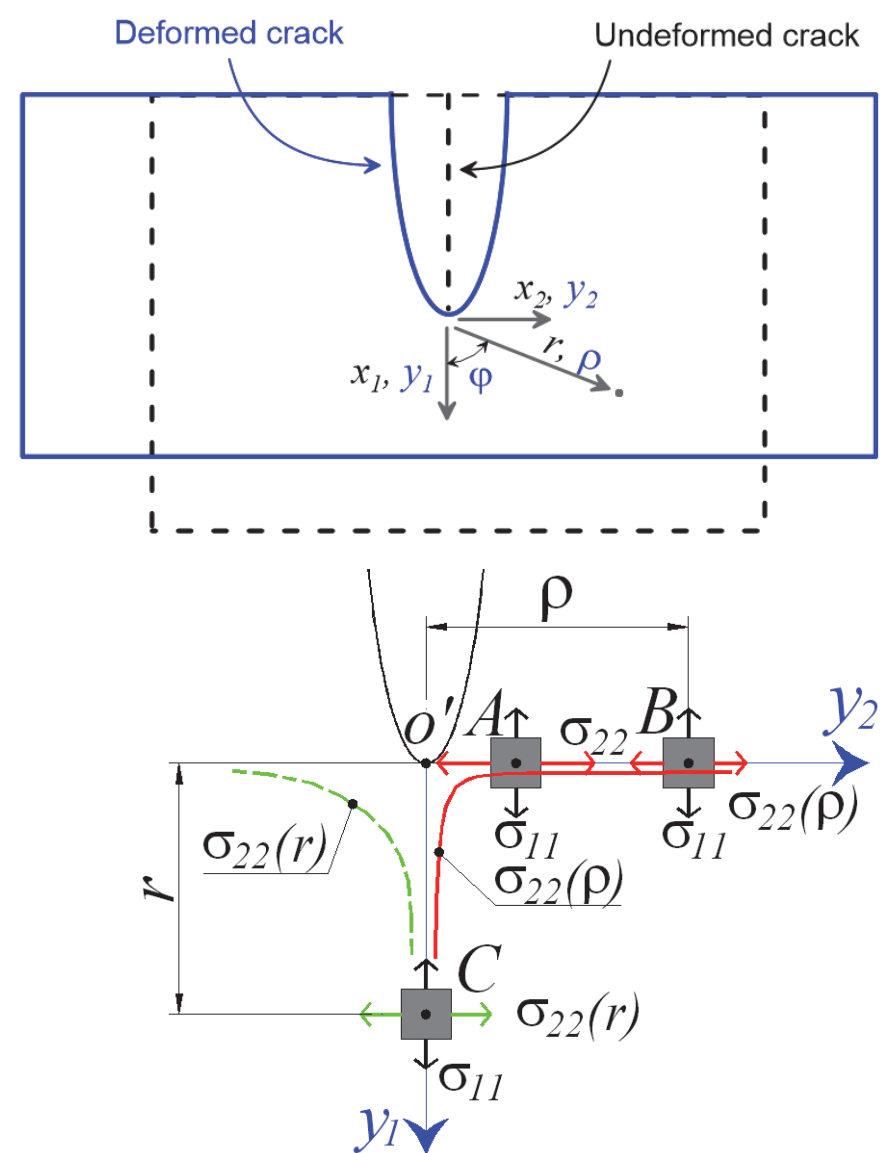

(a)

Figure 2: (a) Undeformed cracked plate and deformed configuration with the related reference systems. (b) Crack tip detail of the stress field. In large deformation, the singularity of the true stress $\sigma_{22}$ is sharper along the $y_{2}$ axis than along the $y_{1}$ axis.

\section{Expected crack path in large deformation}

From Eqs (6),(7) it can be remarked that the stress component $\sigma_{22}$ along the deformed parabolic crack profile $(\varphi=\pi / 2)$ has a singularity -2 (i.e. $\rho^{-2}$ ), while the same stress component has a singularity -1 (i.e. $r^{-1}$ ) ahead of the crack tip $(\varphi=0)$. This different singularity can trigger the appearance of a secondary crack, departing from the blunted deformed one (crack tip splitting), leading to a tortuous crack path or to a rough crack profile. In other words, the singularity of the true stress $\sigma_{22}$ is sharper along the $y_{2}$ axis than along the $y_{1}$ one (see Fig. 2); thus the material tends easily to break apart close to the crack tip along $y_{2}$, leading to the appearance of secondary cracks, responsible for curved crack paths. 
Finally, the rate-dependence of failure in elastomers is a well-known phenomenon and has been explained through the internal energy dissipation mechanism by assuming that the chains bond lifetime depends on the applied stress level $\sigma$ [21]. A slower strain rate allows the material to easily flow in time, while a faster rate entails a more elastic behavior. The experimental outcomes have shown that the crack branching easily occurs for slower strain rates.

\section{EXPERIMENTAL TESTS}

7 he mechanical response under tension of a pre-cracked elastomeric sheet, made of a common silicone polymer, has been experimentally investigated. The evolution of the deformation and of the crack path have been controlled and monitored through the "contact-free" Digital Image Correlation (DIC) measurement technique. The specimens, having width equal to $W=112 \mathrm{~mm}$, are characterized by an initial elastic modulus of about $E=1.12 \mathrm{MPa}$ and Poisson's ratio $v \cong 0.42$, whereas samples' geometric characteristics are shown in Tab.1. The ratio $2 L / W$ is assumed to be sufficiently high to ensure a uniaxial stress state in the central part of the sample, by limiting the boundary effects. The dimensionless small deformation stress intensity factors $K_{\mathrm{I}}^{*}=K_{\mathrm{I}} /\left(\sigma_{y, 0} \sqrt{\pi a}\right)$ are also reported in Tab. 1. The tests have been conducted by applying a controlled displacement to the edge of the plate placed at $Y=+L$, while the other has been kept fixed; three different strain rates have been adopted, namely: $\dot{\varepsilon}_{1}=9.615 \cdot 10^{-3} \mathrm{~s}^{-1}, \quad \dot{\varepsilon}_{2}=5.769 \cdot 10^{-3} \mathrm{~s}^{-1}$ and $\dot{\varepsilon}_{3}=1.603 \cdot 10^{-4} \mathrm{~s}^{-1}(\dot{\varepsilon}=\dot{d} /(2 L), \dot{d}$ being the applied displacement rate), in order to investigate the effect of the deformation velocity on the mechanical response of the cracked plates.

\begin{tabular}{ccccc}
\hline Spec. No. & $2 a(\mathrm{~mm})$ & $t(\mathrm{~mm})$ & $2 a / W(--)$ & $K_{\mathrm{I}}^{*}(---)$ \\
C2a & 20 & 2.75 & 0.179 & 1.019 \\
C2b & 20 & 2.85 & 0.179 & 1.019 \\
C2c & 20 & 2.75 & 0.179 & 1.019 \\
C3a & 30 & 3.00 & 0.268 & 1.045 \\
C3b & 30 & 3.00 & 0.268 & 1.045 \\
C3c & 30 & 2.60 & 0.268 & 1.045 \\
C4a & 40 & 2.75 & 0.357 & 1.084 \\
C4b & 40 & 1.80 & 0.357 & 1.084 \\
C4c & 40 & 2.00 & 0.357 & 1.084 \\
\hline
\end{tabular}

Table 1: Geometric characteristics of the tested specimens and related dimensionless SIF, $K_{\mathrm{I}}^{*}$. The symbol Cn $\alpha(\mathrm{n}=2,3,4,5 ; \alpha=\mathrm{a}$, b, c) refers to plates with different crack lengths tested at strain rates: $\alpha=$ a, strain rate $\dot{\varepsilon}_{1} ; \alpha=$ b, strain rate $\dot{\varepsilon}_{2} ; \alpha=$ c, strain rate $\dot{\varepsilon}_{3}$.

The true stress vs stretch behavior of the examined material under tension is reported in Fig. 3a; the true stress has been obtained from the nominal stress by exploiting the incompressibility assumption. Both the neo-Hookean model and the Gent model are also reported to underline which is the best fitting theoretical model. In Fig. 3b, the variation of the Poisson's ratio measured experimentally vs the stretch value and the corresponding curve for a perfectly incompressible material are displayed; in particular, the theoretical Poisson's ratio vs stretch for an isochoric deformation is expressed as $v=-\left(\lambda^{-1}-1\right) /\left(\lambda^{2}-1\right)$ [22]. From the above-mentioned mechanical tests, it can be concluded that the material behaves very close to the predictions deduced according to the Gent model, and is nearly incompressible.

From the tested material, the mechanical parameters have been found to be $E=1.5 \mathrm{MPa}$ and $J_{m}=2.35$. 

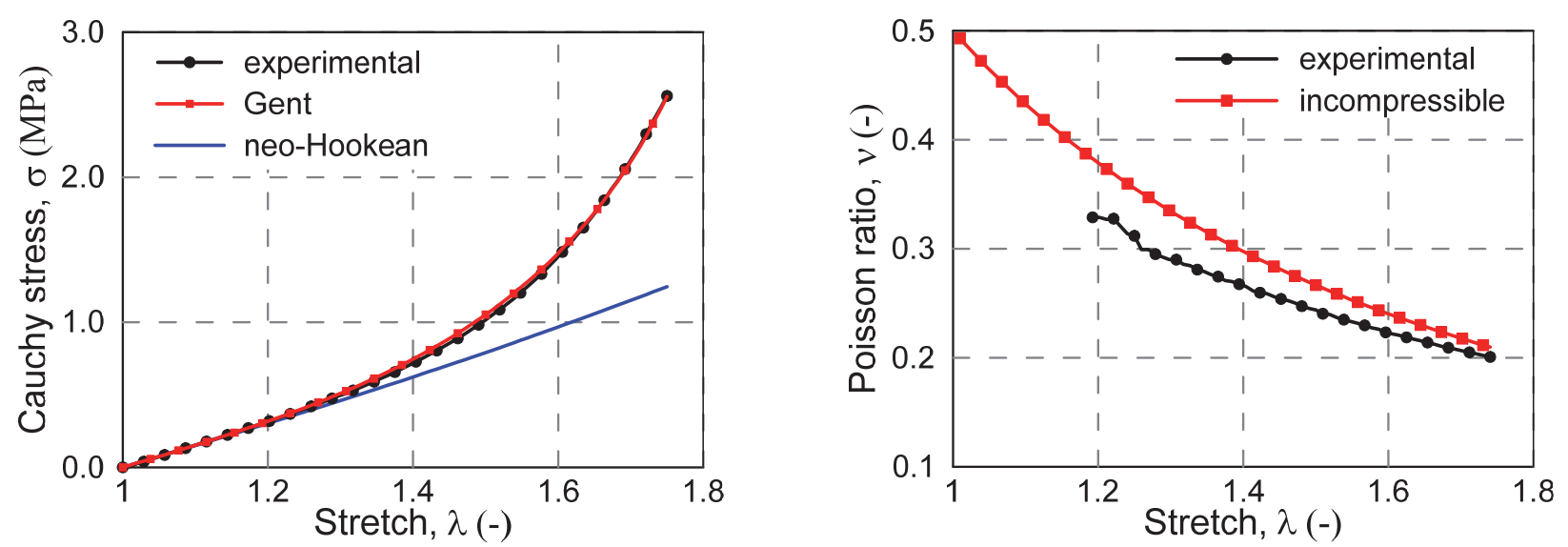

Figure 3: (a) True stress vs stretch for the examined material. (b) Variation of the Poisson's ratio with the deformation. Experimental and theoretical values are reported.

In Fig. 4 the crack tip splitting phenomenon is shown at the incipient failure for the specimens C2b, C3b and C4b, while the whole crack paths after failure are depicted in Fig. 4 for the three strain rates adopted, $\dot{\varepsilon}_{1}, \dot{\varepsilon}_{2}, \dot{\varepsilon}_{3}$.
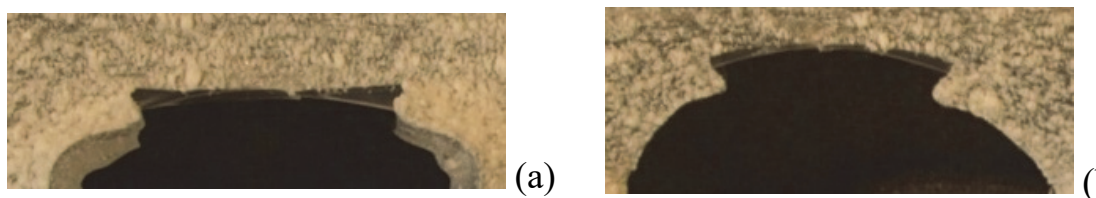

(b)

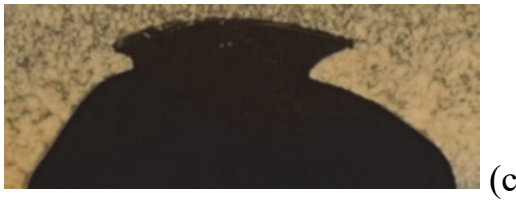

Figure 4: Crack tip splitting at the incipient failure for specimens (a) C2b, (b) C3b and (c) C4b.
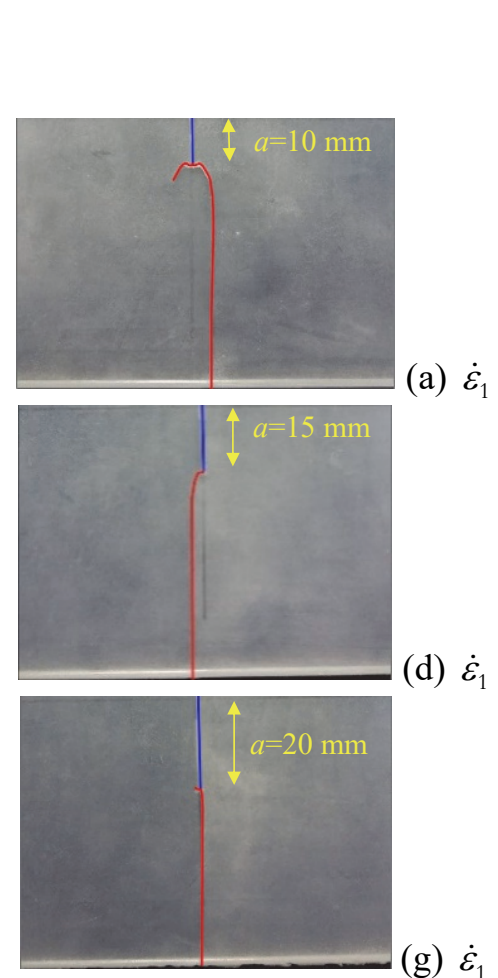

(d) $\dot{\varepsilon}_{1}$

(g) $\dot{\varepsilon}_{1}$

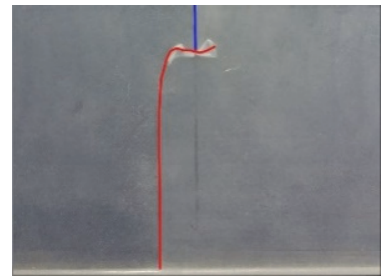

(b) $\dot{\varepsilon}_{2}$
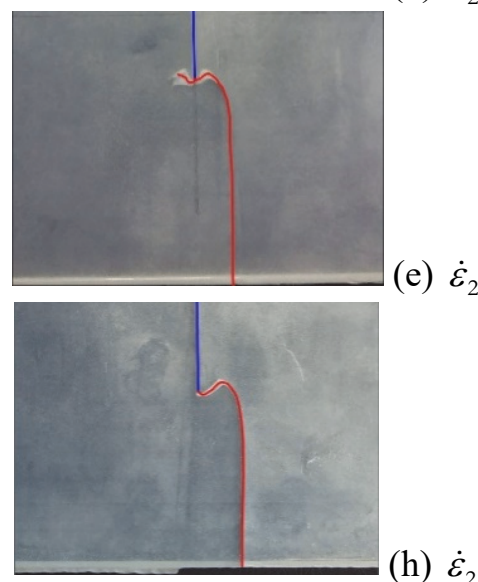

(e) $\dot{\varepsilon}_{2}$

(h) $\dot{\varepsilon}_{2}$
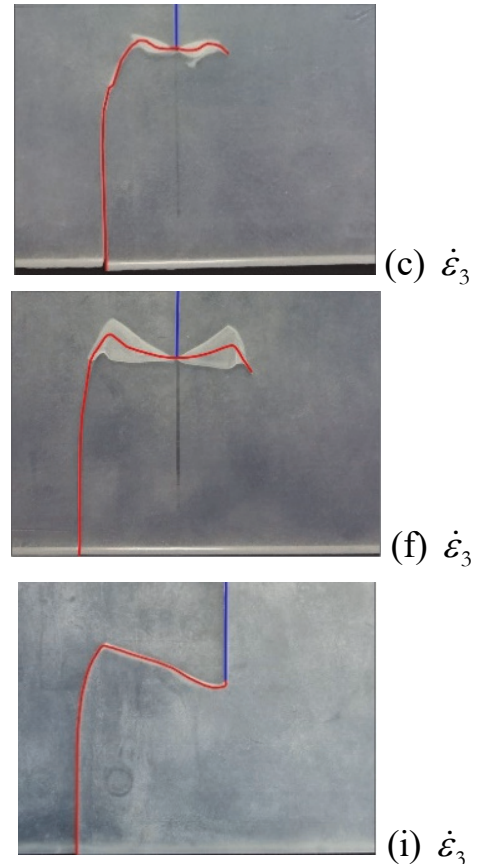

Figure 5: Crack paths for specimens (a) C2a, (b) C2b, (c) C2c, (d) C3a, (e) C3b, (f) C3c, (g) C4a, (h) C4b and (i) C4c (only half of each specimen corresponding to $Y>0$, see Fig. 1, is shown). The initial crack (blue straight line) grows as is indicated by the red line. 
Fig. 5 shows the crack paths obtained for the different cracked samples by changing the initial crack length $2 a=20,30,40 \mathrm{~mm}$ and the applied strain rate (only half of the specimen is shown). It can be appreciated that the resulting crack paths, observed after the final failure of the specimen, are only slightly influenced by the initial crack size, while the strain rate plays a crucial role. It's worth noting that, because of the testing imperfections, the final failure pattern in the specimens usually appeared to be symmetric with respect to the $Y$ axis, while in some cases the crack propagated only in one side of the sheet. In these latter cases, only the cracks half of the specimens have been shown (Fig. 5).

The observed results allow us to obtain a physical interpretation to the way how the crack behaves: under a fast deformation, the material behaves in an elastic way, so that the theoretical stress singularities shown in Eq. (6) are fulfilled. On the other hand, a low strain rate allows the material to damage locally in preferential directions, according to the severity of the stress component singularity, leading to a more marked crack kinking. In this case, the crack initially tends to propagate practically in almost pure Mode II, i.e. in a direction normal to the crack line. From the crack tip stress field viewpoint (see Eqs (6), (7) ), when the material deforms elastically - such as in the small deformation regime - the stress component $\sigma_{22}$ along the deformed parabolic crack profile $(\varphi=\pi / 2)$ is more $\operatorname{singular}\left(\rho^{-2}\right)$ than along the crack symmetry axis $\left(\varphi=0, r^{-1}\right)$. This fact justifies the crack tip split taking place at the crack tip at the very early stage of the deformation process. The numerical analysis reported in Krishnan et al. [23] shows that a concentration of shear deformation exists near the crack tips, especially when the material display a less pronounced strain hardening behavior, such as happens in the case of low strain rates. This justifies the more evident persistence of the Mode II deformation in cases where the material is subjected to low strain rates (Fig. 5c, f, i) with respect to the cases characterized by high strain rates (Fig. 5a, d, g).

\section{CONCLUSIONS}

I $\mathrm{n}$ the present paper, the crack behavior of soft cracked plates has been examined. The crack tip stress field under large displacements as well as the rate-dependent behavior have been considered. The remarkable result from the literature that the singularity of the true stress in large deformation is quite different from the one according to the LEFM theory has been adopted. Moreover, the different order of singularity of the stress components in large deformation gives rise to crack tip splitting and, consequently, curved crack paths develop even under remote pure Mode I loading. This behavior is enhanced at slow strain rates (see the curved crack paths in Fig. 3c, f, i), while faster strain rates have a lower effect in terms of promoting such a weird crack growth (see Fig. 3a, d, g). In fact, because of the existence of the strain rate effect that typically arises in this class of materials, the purely linear behavior is recovered only when the strain rate is sufficiently small, while a more severe crack tilting is observed (with a consequent curved crack path) thanks to the more pronounced damage arising locally in preferential directions according to the severity of the stress component singularity. In the latter case, the crack tends to propagate seemingly in pure Mode II, i.e. in a direction almost normal to the crack line.

Moreover, the observed crack paths deviate from the pure Mode I propagation irrespective of the initial crack length. The experimental results have shown that the crack tip singular stress field arising in highly deformable materials can lead to complex curved crack paths which cannot be predicted by using the standard LEFM approach.

\section{REFERENCES}

[1] Treloar, L.R.G. (1975). Physics of Rubber Elasticity, Oxford University Press .

[2] Doi, M. (2013). Soft Matter Physics. Oxford: Oxford Univ. Press.

[3] Chen, D., Yoon, J., Chandra, D., Crosby, A.J., Hayward, R.C. (2014). Stimuli-responsive buckling mechanics of polymer films. J. Polym. Sci., part B: Pol. Phys, 52, pp. 1441-1461. DOI: 10.1002/polb.23590.

[4] Roland, C.M. (2006). Mechanical behavior of rubber at high strain rates. Rubber Chem. Tech., 79(3), pp. 429-459. DOI: $10.5254 / 1.3547945$.

[5] Bergström, J.S., Boyce, M.C. (2016). Constitutive modelling of the large strain time-dependent behaviour of elastomers. J. Mech. Phys. Sol., 46, pp. 931-954. DOI: 10.1016/S0022-5096(97)00075-6.

[6] Brighenti, R., Vernerey, F.J., Artoni, F. (2017). Rate-dependent failure mechanism of elastomers. J. Mech. Sci., 130, pp. 448-457. DOI: 10.1016/j.ijmecsci.2017.05.033.

[7] Candau, N., Laghmach, R., Chazeau, L., Chenal, J-M., Gauthier, C., Biben, T., Munch, E. (2014). Strain-induced crystallization of natural rubber and cross-link densities heterogeneities. Macromolecules, 47(16), pp. 5815-5824. DOI:10.1021/ma5006843. 
[8] Flory, P.J. (1989). Statistical mechanics of chain molecules. Cincinnati (Ohio): Hanser-Gardner.

[9] de Gennes, P.G. (1971). Reptation of a polymer chain in presence of fixed obstacles. J. Chem. Physics, 55, pp. 572579. DOI: $10.1063 / 1.1675789$.

[10] Treloar, L.R. (1973). The elasticity and related properties of rubbers. Rep. Prog. Phys., 36, pp. 755-826. DOI: 10.1088/0034-4885/36/7/001.

[11] Trapper, P., Volokh, K.Y. (2008). Cracks in rubber. J. Sol. Struct., 45, pp. 6034-6044. DOI: 10.1016/j.ijsolstr.2008.07.016.

[12] Brighenti, R., Carpinteri, A., Artoni, F. (2017). Defect sensitivity to failure of highly deformable polymeric materials. Theor. Appl. Fract. Mech., 88, pp. 107-116. DOI: 10.1016/j.tafmec.2016.12.005.

[13] Gent, A.N. (1996). Adhesion and strength of viscoelastic solids. Is there a relationship between adhesion and bulk properties?, Langmuir 12, pp. 4492-4496. DOI: 10.1021/la950887q.

[14] Lev, Y., Volokh K.Y. (2016). On cavitation in rubberlike materials. J. App. Mech. 83(4), 0044501-1-4. DOI: $10.1115 / 1.4032377$.

[15] Knowles, J.K., Sternberg, E. (1973). An asymptotic finite deformation analysis of the elastostatic field near the tip of a crack. J. Elast., 3, pp. 67-107. DOI: 10.1007/BF00049265.

[16] Gao, Y.C., Jin, M., Dui, G.S. (2008). Stresses, singularities, and a complementary energy principle for large strain elasticity, Appl. Mech. Rev. 61, 030801. DOI: 10.1115/1.2909715.

[17] Boyce, M.C., Arruda, E.M. (2000). Constitutive models of rubber elasticity: a review, Rubber chemistry and technology, 73, pp. 504-523. DOI: $10.5254 / 1.3547602$.

[18] Knowles, J.K., Sternberg, E. (1973). An asymptotic finite deformation analysis of the elastostatic field near the tip of a crack, J. Elast., 3, pp. 67-107. DOI: 10.1007/BF00045816.

[19] Williams, M. (1957). On the stress distribution at the base of a stationary crack. J. Appl. Mech., 24, pp. 109-114. DOI: $10.1115 / 1.3640470$.

[20] Long, R., Krishnan, V.R., Hui, C.Y. (2011). Finite strain analysis of crack tip fields in incompressible hyperelastic solids loaded in plane stress. Mech. Phys. Sol., 59, pp. 672-695. DOI: 10.1016/j.jmps.2010.12.005.

[21] Hui, C-Y., Tang, T., Lin, Y-Y, Chaudhury, M.K. (2004). Failure of elastomeric polymers due to rate dependent bond rupture. Langmuir, 20, pp. 6052-6064. DOI: 10.1021/la0356607.

[22] Beatty, M. F., Stalnaker, D. O. (1986). The Poisson function of finite elasticity. Journal of applied mechanics, 53(4), pp. 807-813. DOI: $10.1115 / 1.3171862$.

[23] Krishnan, V.R., Hui, C-Y., Long, R., (2008). Finite strain crack tip fields in soft incompressible elastic solids. Langmuir, 24(24), pp. 14245-14253. DOI: 10.1021/la802795e. 